\title{
MEMBERS OF THE COUNCIL
}

\section{3}

\section{OfFICERS}

E. B. Van Vleck, President.

M. W. Haskell,

B. O. Peirce,

\} Vice-Presidents.

F. N. Cole, Secretary.

J. H. TANNER, Treasurer.

D. E. SмIтH, Librarian.

F. N. Cole,

E. W. BRown, VIRGIL SNYDER,

Ex-Presidents

J. H. Van Amringe, Emory McClintock, G. W. HILI,
R. S. WOODWARD, ELIAKIM H. MOORE, T. S. Fiske,
W. F. OsGOOD, H. S. WhiTe, Maxime Bôcher.

\section{Elected Members}

To Serve until December, 1913
H. F. BLIChFELDT,
C. J. KEYSER,
J. L. CoOLIDGE,
J. W. Young.

To Serve until December, 1914
A. B. СовLE,
E. W. Davis,
Oswald Veblen, E. B. Wilson.

To Serve until December, 1915

F. C. FerRy,

R. C. Maclaurin,

W. B. Ford,

J ACOB Westlund.

Editorial Committee of the transactions

Maxime Bôcher,

H. S. WhiTE,

L. E. Dickson 